\title{
Acute Kidney Injury in Patients with the Coronavirus Disease 2019: A Multicenter Study
}

\author{
Xiaoyang Cui ${ }^{a} \quad$ Xin $\mathrm{Yu}^{\mathrm{a}}$ Xiaojing Wu ${ }^{\mathrm{a}}$ Linna Huang ${ }^{\mathrm{a}}$ Ye Tian ${ }^{\mathrm{a}}$ \\ Xu Huang $^{\mathrm{a}}$ Zeyu Zhang $^{\mathrm{a}}$ Zhenshun Cheng $^{\mathrm{b}}$ Qiang Guo $^{c} \quad$ Yi Zhang $^{\mathrm{a}}$ \\ Ying Cai ${ }^{a}$ Qingyuan Zhan ${ }^{a}$ \\ aDepartment of Pulmonary and Critical Care Medicine, Center of Respiratory Medicine, \\ National Clinical Research Center for Respiratory Diseases, Institute of Respiratory \\ Medicine, China-Japan Friendship Hospital, Beijing, PR China; ${ }^{b}$ Department of Pulmonary \\ and Critical Care Medicine, Zhongnan Hospital of Wuhan University, Wuhan, PR China; \\ 'Department of Critical Care Medicine, The First Affiliated Hospital of Soochow University, \\ Suzhou, PR China
}

\section{Keywords}

Acute kidney injury · Coronavirus disease $2019 \cdot$ Extrapulmonary complications

\begin{abstract}
Introduction: Severe acute respiratory viral infections are frequency accompanied by multiple organ dysfunction, including acute kidney injury (AKI). In December 2019, the coronavirus disease 2019 (COVID-19) outbreak began in Wuhan, Hubei Province, China, and rapidly spread worldwide. While diffuse alveolar damage and acute respiratory failure are the main features of COVID-19, other organs may be involved, and the incidence of AKI is not well described. We assessed the incidence and clinical characteristics of AKI in patients with laboratory-confirmed COVID-19 and its effects on clinical outcomes. Methods: We conducted a multicenter, retrospective, observational study of patients with COVID-19 admitted to two general hospitals in Wuhan from 5 January 2020 to 21 March 2020. Demographic data and information on organ dysfunction were collected daily. AKI was defined according to the KDIGO clinical practice guidelines. Early and late AKI were defined as AKI occurring within $72 \mathrm{~h}$ after admission or after 72 h, respectively. Results: Of the 116 patients, AKI developed in 21 (18.1\%) patients. Among them, early and late AKI were found in 13 (11.2\%) and 8 (6.9\%) patients, respectively. Compared with patients without AKI, patients with AKI had more severe organ dysfunction, as indicated by a higher level of disease severity status, higher sequential organ failure assessment (SOFA) score on admission, an increased prevalence of shock, and a higher level of respiratory support. Patients with AKI had a higher SOFA score on admission ( $4.5 \pm 2.1$ vs. 2.8 \pm 1.4, OR $1.498,95 \% \mathrm{Cl} 1.047-2.143$ ) and greater hospital mortality (57.1\% vs. $12.6 \%$, OR 3.998,
\end{abstract}


95\% $\mathrm{Cl} 1.088-14.613)$ than patients without AKI in both the univariate and multivariate analyses. Patients with late AKI, but not those with early AKI, had a significantly prolonged length of stay (19.6 vs. 9.6 days, $p=0.015$ ). Conclusion: Our findings show that admission SOFA score was an independent risk factor for AKI in COVID-19 patients, and patients with AKI had higher in-hospital mortality. Moreover, AKI development after $72 \mathrm{~h}$ of admission was related to prolonged hospitalization time.

(C) 2020 The Author(s)

Published by S. Karger AG, Basel

\section{Introduction}

In December 2019, an outbreak of a pneumonia of unknown cause occurred in Wuhan, Hubei Province, China. By 7 January 2020, Chinese scientists isolated a novel coronavirus, named SARS-CoV-2, previously known as 2019-nCoV [1], from human airway epithelial cells. On 30 January 2020, the World Health Organization (WHO) officially declared the coronavirus disease 2019 (COVID-19) epidemic as a public health emergency of international concern. The clinical symptoms of COVID-19 patients include fever, cough, and fatigue, and a small population of patients exhibit gastrointestinal symptoms. The elderly and people with underlying diseases are susceptible to infection and prone to serious outcomes, which may be associated with acute respiratory distress syndrome and cytokine storm [2, 3]. Previous studies have demonstrated that severe acute respiratory viral infections are accompanied by multi-organ dysfunction, which may contribute to death [4]. In particular, acute kidney injury (AKI) is a common, serious complication in critically ill patients, which may result in increased mortality, longer hospital stays, and higher medical costs [5]. Studies of patients with COVID-19 also demonstrated that, in addition to pneumonia, nonpulmonary organ impairment can be seen, including impairment of the liver, cardiovascular system, and kidneys. The aim of this study was to evaluate the incidence, risk factors, and impact on mortality of AKI in critically ill patients with COVID-19.

\section{Materials and Methods}

\section{Study Design and Data Collection}

We conducted a retrospective multicenter study enrolling two cohorts of adult inpatients (>18 years of age) from Zhongnan Hospital of Wuhan University and Tongji Hospital of Huazhong University of Science and Technology Sino-French New City Branch from 5 January 2020, (i.e., when the first patient was admitted) to 21 March 2020. The two hospitals, located in Wuhan, Hubei Province, are teaching and general hospitals. In early January, these two hospitals were designated to treat COVID-19 patients. We continuously enrolled patients diagnosed with COVID-19 by respiratory specimens using real-time RT-PCR methods.

Two physicians (Y.C. and Y.Z.) extracted the epidemiological, demographic, clinical, laboratory, treatment, and outcome data from the electronic medical records system using a standard case report form. After finishing the form, a researcher (Y.T.) adjudicated any differences in interpretation between the two primary reviewers. Patients without the laboratory confirmation of SARS-CoV-2 infection were excluded.

\section{Laboratory Procedures}

Throat swab specimens for SARS-CoV-2 RNA were detected by health institutions using real-time RT-PCR before or soon after admission to the hospital. All patients completed routine blood examinations, coagulation profiles, and serum biochemical tests (including 


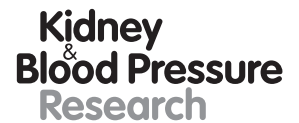

Kidney
Blood Pressure
Research

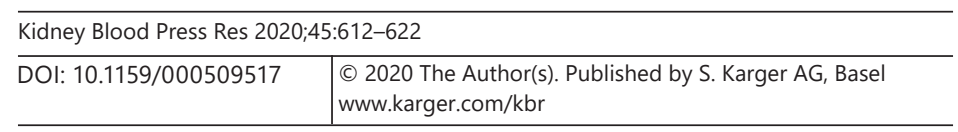

Cui et al.: Acute Kidney Injury in Patients with COVID-19

renal and liver function, and electrolytes) on admission or the next morning. Myocardial enzymes, creatine kinase, lactate dehydrogenase, interleukin-6, serum ferritin, erythrocyte sedimentation rate, and high-sensitivity C-reactive protein were tested according to the patient condition. The upper limit of normal for serum creatinine was $104 \mu \mathrm{mol} / \mathrm{L}$. The frequency of examination was daily or second daily, as determined by the treating physician.

\section{Definitions}

Respiratory comorbidities included chronic obstructive lung disease or asthma, pleural effusion, tuberculosis infection or nontuberculous mycobacteria infection, bronchiectasis, and lung cancer. Cardiovascular comorbidities included coronary heart disease, hypertension, arrhythmia, heart failure, and pericardial effusion. The immunosuppressed state included the following: corticosteroid or immunosuppressant use within 90 days unrelated to COVID-19, seropositive for human immunodeficiency virus, malignant tumor, and patients receiving radiation therapy or chemotherapy for an underlying malignancy within 90 days. Fever was defined as an axillary temperature of at least $37.3^{\circ} \mathrm{C}$. AKI was diagnosed according to the KDIGO clinical practice guidelines [6]. If the patient had not previously had a history of chronic kidney disease (CKD) and the serum creatinine on admission was $26.5 \mu \mathrm{mol} / \mathrm{L}$ more than the upper limit $(104 \mu \mathrm{mol} / \mathrm{L})$, the patient was considered to have AKI. The stage of AKI was determined using the peak serum creatinine level after AKI detection, with increases of 1.5-1.9, 2.0-2.9, and 3 or more times baseline being defined as AKI stage 1, 2, and 3, respectively.

Early AKI was defined as AKI occurring within $72 \mathrm{~h}$ of hospital admission. Patients who developed AKI later than $72 \mathrm{~h}$ after hospital admission were classified into the late AKI group. The illness severity of COVID-19 was defined according to the Chinese management guideline for COVID-19 (version 7.0) [7]. Each patient was assigned the sequential organ failure assessment (SOFA) score on admission [8]. Shock was defined as arterial systolic blood pressure less than $90 \mathrm{~mm} \mathrm{Hg}$, mean arterial pressure less than $60 \mathrm{~mm} \mathrm{Hg}$, or use of norepinephrine at any dosage to maintain systolic blood pressure at $90 \mathrm{~mm} \mathrm{Hg}$ or more or mean arterial pressure at $60 \mathrm{~mm} \mathrm{Hg}$ or more. Fluid balance was calculated as the difference between fluid input and fluid output. Fluid input included all fluids infused by intravenous or enteral routes. Fluid output included urine output, volume of fecal matter, and fluid loss from drains.

\section{Statistical Analysis}

Clinical data were compared between patients with and without AKI, as well as between patients with early and late AKI. Categorical variables were analyzed using the $\chi^{2}$ test or Fisher's exact test, and continuous variables were analyzed using Student's $t$ test or the MannWhitney $U$ test. After testing the distribution of continuous variables, normally distributed variables were presented as the mean $\pm \mathrm{SD}$, and non-normally distributed variables were presented as the median (interquartile range). Multivariate analysis was performed to evaluate risk factors for AKI and clinical outcomes, which were expressed as the odds ratio (OR) and its 95\% confidence interval (CI). A two-sided $p$ value less than 0.05 was considered statistically significant. SPSS 18.0 (SPSS, Chicago, IL, USA) was used for all statistical analyses.

\section{Results}

General Characteristics

During the observation period, 158 patients were continuously admitted to the two centers. Forty-two patients were excluded because of a clinical diagnosis without nucleic acid test results. The characteristics of the 116 patients included are shown in Table 1. 
Table 1. Patient characteristics and clinical outcomes of patients with COVID-19

\begin{tabular}{|c|c|c|c|}
\hline & AKI $(n=21)$ & Non-AKI $(n=95)$ & $p$ value \\
\hline \multicolumn{4}{|l|}{ Prehospital information } \\
\hline Age, years & $61.05 \pm 12.9$ & $58.58 \pm 14.6$ & 0.477 \\
\hline Sex (male) & $12(57.1)$ & $54(56.8)$ & 0.122 \\
\hline BMI & $23.9 \pm 6.5$ & $23.1 \pm 2.5$ & 0.449 \\
\hline \multicolumn{4}{|l|}{ Comorbidity } \\
\hline Respiratory diseases & $4(19.0)$ & $10(10.5)$ & 0.475 \\
\hline Cardiovascular disease & $10(47.6)$ & $38(40)$ & 0.692 \\
\hline Hypertension & $9(42.9)$ & $29(30.5)$ & 0.405 \\
\hline Diabetes mellitus & $2(9.5)$ & $26(27.4)$ & 0.202 \\
\hline Chronic kidney disease & $1(4.8)$ & $4(4.2)$ & 1 \\
\hline \multicolumn{4}{|l|}{ Time from symptom onset to hospital } \\
\hline admission, days & $10.81 \pm 12.30$ & $10.44 \pm 7.85$ & 0.894 \\
\hline Immunosuppressed & $2(9.5)$ & $5(5.3)$ & 0.814 \\
\hline Antiviral use before admission to the hospital & $8(38)$ & $45(47.4)$ & 0.596 \\
\hline \multicolumn{4}{|l|}{ In-hospital situation } \\
\hline \multicolumn{4}{|l|}{ Symptoms } \\
\hline Fever & $21(100)$ & $78(82.1)$ & 0.062 \\
\hline Cough & $17(81)$ & $62(65.3)$ & 0.163 \\
\hline Myalgia & $5(23.8)$ & $24(25.3)$ & 0.889 \\
\hline Diarrhea & $4(19)$ & $26(27.4)$ & 0.431 \\
\hline \multicolumn{4}{|l|}{ Laboratory findings } \\
\hline WBC, $\times 10^{9} / \mathrm{L}$ & $6.88(4.83-8.59)$ & $5.43(4.03-6.83)$ & 0.448 \\
\hline $\mathrm{LYM}, \times 10^{9} / \mathrm{L}$ & $0.77(0.49-0.92)$ & $0.83(0.55-1.27)$ & 0.224 \\
\hline $\mathrm{CK}, \mathrm{U} / \mathrm{L}$ & $165.15(70.75-1,004.5)$ & $81.00(47.75-160.00)$ & $0.002^{*}$ \\
\hline NT-proBNP, pg/mL & $471.5(231.00-1,836.27)$ & $253.0(91.00-633.00)$ & 0.683 \\
\hline D-Dimer, $\mu \mathrm{g} / \mathrm{L}$ & $0.53(0.21-2.58)$ & $0.53(0.26-1.56)$ & 0.912 \\
\hline hs-CRP, mg/L & $57.50(35.90-144.33)$ & $57.90(15.00-104.60)$ & 0.500 \\
\hline $\mathrm{ESR}, \mathrm{mm} / \mathrm{h}$ & $37.00(13.75-73.25)$ & $39.00(22.00-60.00)$ & 0.975 \\
\hline Ferritin, $\mu \mathrm{g} / \mathrm{L}$ & $515.40(372.58-2,639.23)$ & $618.40(321.34-1,486.95)$ & 0.669 \\
\hline IL-6, pg/mL & $46.46(12.20-89.04)$ & $22.10(5.88-52.18)$ & 0.938 \\
\hline \multicolumn{4}{|l|}{ Disease severity status } \\
\hline General & $4(19)$ & $37(38.9)$ & 0.084 \\
\hline Severe & $6(28.6)$ & $31(32.6)$ & 0.718 \\
\hline Critical & $11(52.4)$ & $26(27.4)$ & $0.026^{*}$ \\
\hline SOFA score & $4.5 \pm 2.1$ & $2.8 \pm 1.4$ & $0.002 *$ \\
\hline \multicolumn{4}{|l|}{ Respiratory support } \\
\hline Ambient air & $1(4.8)$ & $22(23.2)$ & 0.107 \\
\hline Nasal cannula & $6(28.6)$ & $47(49.5)$ & 0.134 \\
\hline HFNC & $3(14.3)$ & $6(6.3)$ & 0.433 \\
\hline NPPV & $2(9.5)$ & $8(8.4)$ & 1 \\
\hline IPPV & $6(28.6)$ & $7(7.4)$ & $0.016^{*}$ \\
\hline ECMO & $3(14)$ & $4(4.2)$ & 0.056 \\
\hline Shock & $10(47.6)$ & $24(25.3)$ & $0.042^{*}$ \\
\hline \multicolumn{4}{|l|}{ Outcomes } \\
\hline Mortality & $12(57.1)$ & $12(12.6)$ & $0.000^{*}$ \\
\hline \multicolumn{4}{|l|}{ Time from admission to death or } \\
\hline \multicolumn{4}{|c|}{$\begin{array}{l}\text { Data are presented as mean } \pm \text { SD, median (IQR), or } n(\%) \text {, as appropriate. WBC, white blood cell count; LYM, lymphocyt } \\
\text { count; CK, creatine kinase; NT-proBNP, N-terminal B-type natriuretic peptide; hs-CRP, high-sensitive C-reactive protein; ESR } \\
\text { erythrocyte sedimentation rate; IL-6, interleukin- } 6 \text {; HFNC, high-flow nasal cannula oxygen therapy; NPPV, noninvasive positiv } \\
\text { pressure ventilation; IPPV, invasive positive pressure ventilation; ECMO, extracorporeal membrane oxygenation. Antiviru } \\
\text { medications included umifenovir, ganciclovir, interferon, lopinavir with ritonavir, oseltamivir, and ribavirin. * } p<0.05 \text { wa } \\
\text { considered significant. }\end{array}$} \\
\hline
\end{tabular}




\section{Kidney \\ Blood Pressure \\ Research}

Table 2. Multivariate analysis of variables and outcomes associated with AKI in patients with COVID-19

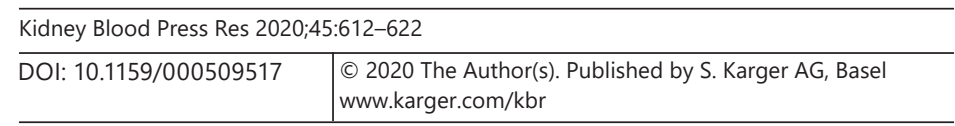

Cui et al.: Acute Kidney Injury in Patients with COVID-19

\begin{tabular}{lll}
\hline & OR $(95 \% \mathrm{CI})$ & $p$ \\
\hline SOFA score & $1.498(1.047-2.143)$ & $0.027^{*}$ \\
Mortality & $3.988(1.088-14.613)$ & $0.037^{*}$ \\
Critical & & 0.338 \\
Shock & & 0.224 \\
IPPV & & 0.795 \\
\hline
\end{tabular}

$* p<0.05$ was considered significant.

Of the 116 patients evaluated in this study, the mean age was 59 years, 55 (47.4\%) were under 60 years of age, and $66(56.9 \%)$ were male. Fourteen $(12 \%)$ of them had underlying respiratory diseases (6 COPD or asthma, 2 pleura effusion, 3 tuberculosis or nontuberculous mycobacteria infection, 2 lung cancer, and 1 bronchiectasis), 48 (41.4\%) had circulatory system diseases (14 coronary heart disease, 38 hypertension, 3 arrhythmia, 1 heart failure, and 1 pericardial effusion), 28 (24.1\%) had diabetes mellitus, and 5 (4.3\%) had CKD. The disease severity status of the patients was as follows: 48 (41.4\%) general, 47 (40.5\%) severe, and $20(17.2 \%)$ critical. Twenty-three (19.8\%) patients did not need oxygen therapy, 53 $(45.7 \%)$ patients used nasal oxygen inhalation, and 9 (7.76\%) patients needed high-flow nasal cannula oxygen therapy. Mechanical ventilation was used in $23(19.8 \%)$ patients; among them, 10 patients needed noninvasive mechanical ventilation, and 13 patients needed invasive mechanical ventilation. Moreover, 7 (6\%) of the 116 included patients needed extracorporeal membrane oxygenation (ECMO) support because of severe respiratory failure. Eighteen patients died, a mortality rate of $15.5 \%$, and the median time from admission to discharge or death was 14 days (IQR 10-22).

\section{Acute Kidney Injury}

AKI developed in 21 (18.1\%) of the 116 patients with COVID-19. The demographic information, prevalence of different comorbidities, time from onset to hospital admission, immunosuppressed state, and symptoms were not significantly different between AKI and non-AKI patients. Compared with patients without AKI, patients with AKI had higher creatine kinase ( 165.15 vs. $81 \mathrm{U} / \mathrm{L}, p=0.002)$ and more severe organ dysfunction, as indicated by a higher level of disease severity status (critical: 52.4 vs. $27.4 \%, p=0.026$ ), higher SOFA score on admission ( $4.5 \pm 2.1$ vs. $2.8 \pm 1.4, p=0.002$ ), a greater presence of shock (47.6 vs. $25.3 \%, p=0.042$ ), and a higher level of respiratory support (mechanical ventilation: 38.1 vs. $15.8 \%$, ECMO: 14 vs. $4.2 \%$ ). Patients with AKI had higher hospital mortality than patients without AKI in both the univariate and multivariate analyses (57.1 vs. $12.6 \%, p=0.000$ ) (Table 2).

Early versus Late AKI

Early AKI was found in 13 patients (11.2\% of all patients), and late AKI was found in 8 patients (6.9\%) (Table 3).

A male predominance was more obvious in the early AKI group (69.2 vs. $37.5 \%, p=$ 0.331). The BMI of the late AKI patients was higher than that of early AKI patients, but the difference was not statistically significant ( $25.6 \pm 3.43$ vs. $22.5 \pm 8.15, p=0.326)$. The comorbidities and laboratory findings were not different between the two groups. Regarding the disease severity status, the patients' condition in the early AKI group was worse than that in the late AKI group (critical: 61.5 vs. $37.5 \%, p=0.534$ ), they had a higher SOFA score on admission ( $5.00 \pm 1.96$ vs. $3.63 \pm 2.07, p=0.142)$, and needed a higher level of respiratory 
Table 3. Subgroup analysis of early and late AKI in patients with COVID-19

\begin{tabular}{|c|c|c|c|}
\hline & Early AKI $(n=13)$ & Late AKI $(n=8)$ & $p$ value \\
\hline \multicolumn{4}{|l|}{ Prehospital information } \\
\hline Age, years & $60.69 \pm 14.19$ & $61.63 \pm 11.30$ & 0.877 \\
\hline Sex (male) & $9(69.2)$ & $3(37.5)$ & 0.331 \\
\hline BMI & $22.5 \pm 8.15$ & $25.6 \pm 3.43$ & 0.326 \\
\hline \multicolumn{4}{|l|}{ Comorbidity } \\
\hline Respiratory diseases & $2(15.4)$ & $2(25)$ & 1 \\
\hline Cardiovascular disease & $7(53.8)$ & $3(37.5)$ & 0.781 \\
\hline Hypertension & $6(46.2)$ & $3(37.5)$ & 1 \\
\hline Diabetes mellitus & $1(7.7)$ & $1(12.5)$ & 1 \\
\hline Chronic kidney disease & 0 & 1 & \\
\hline Immunosuppressed & $2(15.4)$ & $0(0)$ & 0.505 \\
\hline Antiviral use before admission to the hospital & $6(46.2)$ & $2(25)$ & 0.612 \\
\hline \multicolumn{4}{|l|}{ In-hospital situation } \\
\hline \multicolumn{4}{|l|}{ Laboratory findings } \\
\hline WBC, $\times 10^{9} / \mathrm{L}$ & $6.67(5.04-12.99)$ & $7.23(3.97-7.87)$ & 0.280 \\
\hline LYM, $\times 10^{9} / \mathrm{L}$ & $0.77(0.42-0.88)$ & $0.78(0.54-0.99)$ & 0.898 \\
\hline D-Dimer, $\mu \mathrm{g} / \mathrm{L}$ & $0.53(0.28-2.89)$ & $0.56(0.19-2.74)$ & 0.667 \\
\hline $\mathrm{ESR}, \mathrm{mm} / \mathrm{h}$ & $35.00(13.50-63.00)$ & $66.00(19.00-82.00)$ & 0.387 \\
\hline $\mathrm{IL}-6, \mathrm{pg} / \mathrm{mL}$ & $40.17(7.64-73.43)$ & $94.07(31.92-169.35)$ & 0.067 \\
\hline \multicolumn{4}{|l|}{ Disease severity status } \\
\hline General & $2(15.4)$ & $2(25.0)$ & 1 \\
\hline Severe & $3(33.3)$ & $3(37.5)$ & 1 \\
\hline Critical & $8(61.5)$ & $3(37.5)$ & 0.534 \\
\hline \multicolumn{4}{|l|}{ Respiratory support } \\
\hline Ambient air & $1(7.7)$ & $0(0)$ & 1 \\
\hline Nasal cannula & $2(15.4)$ & $4(50)$ & 0.227 \\
\hline HFNC & $1(7.7)$ & $2(25)$ & 0.647 \\
\hline NPPV & $2(15.4)$ & $0(0)$ & 0.505 \\
\hline IPPV & $5(38.5)$ & $1(12.5)$ & 0.434 \\
\hline ECMO & $2(15.4)$ & $1(12.5)$ & 1 \\
\hline Shock & $5(38.5)$ & $3(37.5)$ & 1 \\
\hline \multicolumn{4}{|l|}{ Outcomes } \\
\hline Mortality & $7(53.8)$ & $5(62.5)$ & 1.000 \\
\hline Time from admission to death or discharge, days & $9.60 \pm 4.14$ & $19.63 \pm 10.78$ & $0.015^{*}$ \\
\hline
\end{tabular}

Data are presented as mean \pm SD, median (IQR), or $n(\%)$, as appropriate. WBC, White blood cell count; LYM, Lymphocyte count; ESR, erythrocyte sedimentation rate; IL-6, interleukin-6; HFNC, high-flow nasal cannula oxygen therapy; NPPV, noninvasive positive pressure ventilation; IPPV, invasive positive pressure ventilation; ECMO, extracorporeal membrane oxygenation. Antivirus medications included in umifenovir, ganciclovir, interferon, lopinavir with ritonavir, oseltamivir, and ribavirin. $* p<0.05$ was considered significant.

support (mechanical ventilation: 53.9 vs. $12.5 \%$, ECMO: 15.4 vs. $12.5 \%$ ). As for the severity stage of AKI between the two groups, the majority of patients in the early AKI group were in stage $1(n=9,69.5 \%)$ whereas half of the patients in the late AKI group were in stage $3(n=$ $4,50 \%$ ). The mean peak serum creatinine was similar in both groups (early vs. late AKI groups: $233 \pm 170$ vs. $204 \pm 109 \mu \mathrm{mol} / \mathrm{L}, p=0.66$ ). Compared with patients with early AKI, patients with late AKI had a significantly prolonged hospital length of stay (19.63 $\pm 10.78 \mathrm{vs}$. $9.6 \pm 4.14$ days, $p=0.015$ ) and higher mortality ( 62.5 vs. $53.8 \%, p=1.000)$. Among the 13 patients with early AKI, 6 patients' renal function improved before discharge or death, while 7 patients' renal function did not improve. 
Table 4. Fluid balance of AKI and non-AKI patients

\begin{tabular}{lccc}
\hline & AKI & Non-AKI & $p$ \\
\hline Fluid balance, $\mathrm{mL} / 24 \mathrm{~h}$ & $457.2 \pm 123.6$ & $182.1 \pm 78.9$ & 0.07 \\
Urine output, $\mathrm{mL} / \mathrm{kg} / \mathrm{h}$ & $0.8 \pm 0.7$ & $1.2 \pm 0.4$ & 0.18 \\
\hline
\end{tabular}

Data are presented as mean \pm SD. Fluid balance was calculated as the difference between the daily fluid input and fluid output.. $p<0.05$ was considered significant.

Fig. 1. Daily fluid balance of the AKI and non-AKI patients. Fluid balance was calculated as the difference between the daily fluid input and fluid output. Data are expressed as mean \pm SEM.

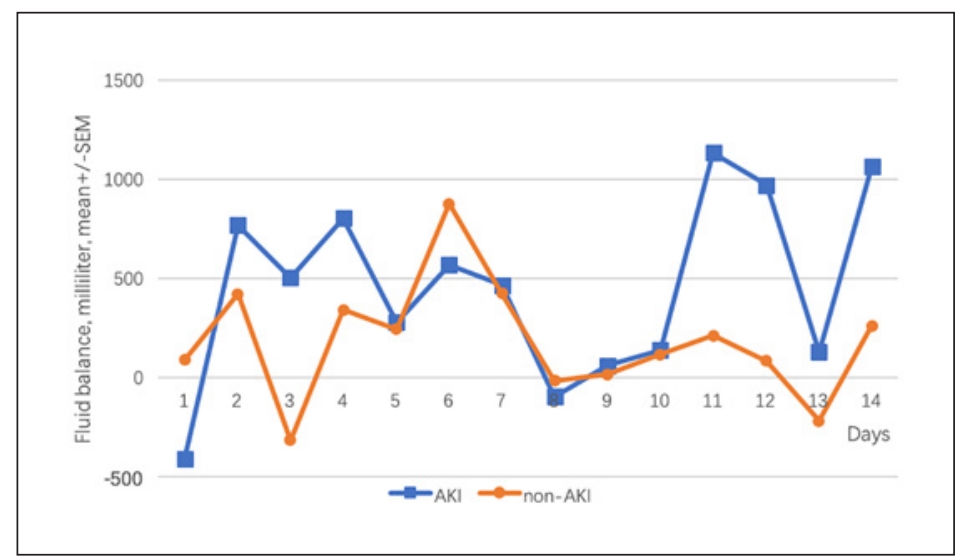

\section{Fluid Balance and AKI}

In our study, $10 \mathrm{AKI}$ patients and 14 non-AKI patients had daily fluid balance recorded. AKI patients had more fluid accumulation than non-AKI patients $(457.2 \pm 123.6$ vs. $182.1 \pm$ $78.9 \mathrm{~mL} / 24 \mathrm{~h}, p=0.07)$ and lesser urine output ( $0.8 \pm 0.7$ vs. $1.2 \pm 0.4 \mathrm{~mL} / \mathrm{kg} / \mathrm{h}, p=0.18)$ (Table 4; Fig. 1). This trend was more prominent in the early and late AKI subgroups. Lateonset AKI patients had significantly more fluid accumulation than early-onset AKI patients $(-157.5 \pm 278.9$ vs. $769.4 \pm 125.2 \mathrm{~mL} / 24 \mathrm{~h}, p=0.007)$ and more severe kidney damage (urine output: $1.0 \pm 0.9$ vs. $0.7 \pm 0.5 \mathrm{~mL} / \mathrm{kg} / \mathrm{h}, p=0.48$ ) (Table 5; Fig. 2).

\section{Discussion}

The present study found an incidence of AKI of 18.1\% among patients admitted with COVID-19 in Hubei Province, China. AKI was generally diagnosed in sicker patients. Most of the patients in the AKI group were critical (52.4\%), had a higher admission SOFA score (4.5 \pm 2.1 ), and had combined shock (47.6\%), while in the non-AKI group only a quarter of patients were critical (27.4\%), the mean admission SOFA score was $2.8 \pm 1.4$, and the incidence of shock was significantly lower (25.3\%). The incidence of AKI in COVID-19 patients varies in published studies. Huang et al. [3] reported 41 COVID-19 patients, among whom $10 \%$ had elevated creatinine ( $>133 \mu \mathrm{mol} / \mathrm{L}$ ) on admission and $7 \%$ had AKI. For critically ill patients in the ICU, the incidence was $23 \%$. Zhou et al. [2] found that AKI occurred in $15 \%$ of patients, of which $50 \%$ occurred in nonsurvivors and $1 \%$ occurred in survivors. A retrospective singlecenter study of 138 hospitalized patients in Zhongnan Hospital of Wuhan University from 1 January 2020 to 28 January 2020 indicated that the incidence of AKI was 3.6\% in total patients and $8.3 \%$ in ICU patients [9]. As our study indicated, the admission SOFA score of the patients 
Table 5. Fluid balance of early AKI and late AKI patients

\begin{tabular}{lccl}
\hline & Early AKI & Late AKI & $p$ \\
\hline Fluid balance, $\mathrm{mL} / 24 \mathrm{~h}$ & $-157.5 \pm 278.9$ & $769.4 \pm 125.2$ & $0.007^{*}$ \\
Urine output, $\mathrm{mL} / \mathrm{kg} / \mathrm{h}$ & $1.0 \pm 0.9$ & $0.7 \pm 0.5$ & 0.48 \\
\hline
\end{tabular}

Data are presented as mean \pm SD. Fluid balance was calculated as the difference between the daily fluid input and fluid output. $* p<0.05$ was considered significant.

Fig. 2. Daily fluid balance of the early AKI and late AKI patients. Fluid balance was calculated as the difference between the daily fluid input and fluid output. Data are expressed as mean \pm SEM.

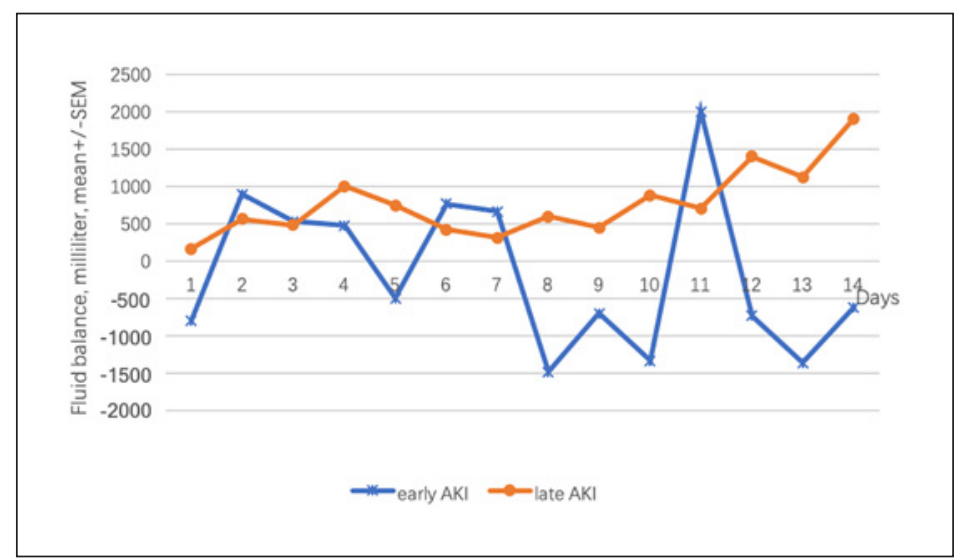

was an independent risk factor for AKI in COVID-19 patients. We speculated the fundamental reason for the different incidence of $\mathrm{AKI}$ in the studies was the difference in the severity of the patients included.

The reason why AKI occurs in COVID-19 patients may be, at least in part, related to the mechanism of SARS-CoV-2 entry into target cells. The first step in SARS-CoV-2 infection is to bind to the host cell receptor and enter the cells. Studies have verified that SARS-CoV-2 uses the SARS-CoV ACE2 receptor for entry $[10,11]$. Therefore, cells with ACE2 expression, such as type II alveolar cells (AT2) in the lung, may act as target cells and may be susceptible to COVID-19 infection. However, it should be noted that ACE2 protein has abundant expression in many different cell types, such as respiratory epithelial cells, myocardial cells, renal tubular epithelial cells, urothelial cells, and gastrointestinal epithelial cells [12]. A clinical implication of this is that, in addition to the lungs, organs expressing ACE2 should be regarded as potentially susceptible to SARS-CoV-2 infection, which may explain the nonrespiratory symptoms observed in COVID-19 patients. Moreover, Farkash et al. [13] offered confirmatory evidence that direct renal infection occurs in the setting of AKI in COVID-19. They performed an autopsy on a single patient who died of COVID-19 and found viral particles in the renal tubular epithelium that were morphologically identical to SARS-CoV-2, and with viral arrays and other features of virus assembly which provided evidence of a direct infection of the kidney by SARS-CoV-2.

Our study revealed that admission SOFA score was independently associated with the development of AKI in COVID-19 patients. This result was consistent with that of a previous study on pandemic influenza A(H1N1) [14].SOFA score indicates the level of organ dysfunction and illness severity [15], which accounts for its association with AKI in the present study. In our study, AKI patients had a significant higher mortality rate after adjusting for admission SOFA score, severity status, respiratory support method, and shock. A previous prospective 


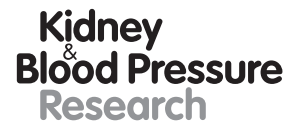

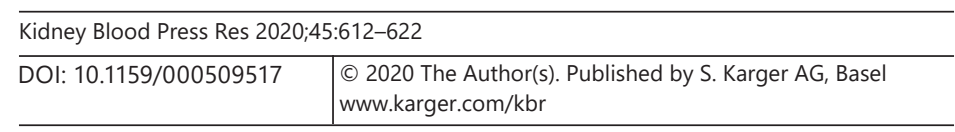

Cui et al.: Acute Kidney Injury in Patients with COVID-19

cohort study of 701 patients with COVID-19 also showed that AKI stage 1 (OR 1.90, 95\% CI 0.76-4.76), stage 2 (OR 3.51, 95\% CI 1.49-8.26), and stage 3 (OR 4.38, 95\% CI 2.31-8.31) were independent risk factors for hospital death [16]. Similar phenomena were observed in SARS patients in 2003, and the mortality rate was significantly higher among patients with SARS and acute renal impairment than among those with SARS and no renal impairment ( 91.7 vs. 8.8\%, $p<0.0001$ ) [17]. Because of the high homology of SARS-CoV-2 and SARS-CoV, the results of this study were similar and consistent with the presentation of renal function injury in SARS.

Previous studies suggested that patients with early and late AKI had different prognosis and may have different pathogenesis $[14,18,19]$. In our subgroup analysis, we also found that the time of onset of AKI characterized two different populations. Patients with late AKI (onset $72 \mathrm{~h}$ after admission) had a higher mortality rate and significantly longer hospitalization time than patients with early AKI. This result is consistent with that presented in the previous study of the 2009 influenza A (H1N1) pandemic. Nin et al. [19] found that patients with early AKI had only slightly and insignificantly higher mortality rates than patients without AKI, whereas patients with late AKI had significantly higher mortality rates than either non-AKI or early AKI patients. This result may be due to early AKI probably being determined, to some extent, by direct cytopathic effects of the virus [10,12, 20], virusinduced specific immunological effector mechanisms [17], virus-induced cytokines or mediator effects [21], and hypovolemic organ hypoperfusion, whereas late AKI generally appeared in combination with sepsis, multiple organ failure, and the use of nephrotoxic agents. In our study, $69.2 \%$ of patients in the early AKI group were in AKI stage 1 , as opposed to $25 \%$ of patients in the late AKI group. This may be another reason for the better prognosis of the early-onset AKI group. However, we should also consider that most of the included patients had no prior baseline serum creatinine record. Therefore, the first serum creatinine level on admission was considered the baseline value. If the patient had no previous history of CKD and the serum creatinine on admission was $26.5 \mu \mathrm{mol} / \mathrm{L}$ more than the upper limit (104 $\mu \mathrm{mol} / \mathrm{L}$ ), the patient was considered to have AKI. This may cause the severity of AKI to be underestimated.

In our study, AKI patients had a trend towards more fluid accumulation and lower urine output than non-AKI patients, with late-onset AKI patients having significantly more fluid accumulation than early-onset AKI patients. There is growing evidence that fluid administration beyond the correction of hypovolemia is associated with AKI [22], longer periods of hospital stay, increased mortality [23], organ dysfunction, and worse clinical outcomes [24]. However, the optimal hydration strategy for patients with COVID-19 cannot be determined from the present data.

Our research was conducted in the early stage of the outbreak in Wuhan, and it has several limitations. First, the two centers (Zhongnan Hospital of Wuhan University and Tongji Hospital of Huazhong University of Science and Technology Sino-French New City Branch) in our study were general and teaching hospitals and they were assigned responsibility by the government for the treatment of patients with severe COVID-19 in January 2020. The patients had relatively severe illness and comorbidities were common, which is likely to have contributed to the high frequency of AKI in this cohort. Second, the small study size limited the capacity for meaningful subgroup analysis. Third, an accurate baseline serum creatinine was not available, which may have led to an overestimate of AKI. Fourth, owing to the lack of data, we could not comment on concomitant medications, urine sediment, or longer-term patient outcomes. 


\section{Conclusion}

The incidence of AKI in COVID-19 patients in our study was 18.1\%. Admission SOFA score was an independent risk factor for AKI in COVID-19 patients, and patients with AKI had higher in-hospital mortality. The development of AKI later in the course of disease was associated with longer hospitalization time.

\section{Acknowledgments}

We thank all the medical staff for their efforts to fight against COVID-19.

\section{Statement of Ethics}

The study protocol was approved by the Ethics Committee of the China-Japan Friendship Hospital and was conducted in full accordance with the principles of the Declaration of Helsinki. All patients provided written informed consent.

\section{Conflict of Interest Statement}

The authors have no conflicts of interest to declare.

\section{Funding Sources}

This study was supported by grants from the Zhejiang University special scientific research fund for COVID-19 prevention and control (2020XGZX008). The funder had no role in the study design, data collection and analysis, decision to publish, or preparation of the manuscript.

\section{Author Contributions}

In the study, X.C., X.Y., and L.H. designed the research project. X.C., X.Y., L.H., Y.T., X.H., Z.Z., X.W., Z.C., Q.G., Y.Z., and Y.C. performed the study and collected the clinical data. X.C. and L.H. analyzed the data. X.C. and X.Y. drafted the manuscript. X.C. and Q.Z. revised and approved the final version of the manuscript.

\section{References}

1 Zhu N, Zhang D, Wang W, Li X, Yang B, Song J, et al.; China Novel Coronavirus Investigating and Research Team. A Novel Coronavirus from Patients with Pneumonia in China, 2019. N Engl J Med. 2020 Feb;382(8):727-33.

2 Zhou F, Yu T, Du R, Fan G, Liu Y, Liu Z, et al. Clinical course and risk factors for mortality of adult inpatients with COVID-19 in Wuhan, China: a retrospective cohort study. Lancet. 2020 Mar;395(10229):1054-62.

3 Huang C, Wang Y, Li X, Ren L, Zhao J, Hu Y, et al. Clinical features of patients infected with 2019 novel coronavirus in Wuhan, China. Lancet. 2020 Feb;395(10223):497-506.

4 Sellers SA, Hagan RS, Hayden FG, Fischer WA 2nd. The hidden burden of influenza: A review of the extrapulmonary complications of influenza infection. Influenza Other Respir Viruses. 2017 Sep;11(5):372-93. 


\section{Kidney \\ Blood Pressure \\ Research}

\begin{tabular}{l|l}
\hline Kidney Blood Press Res 2020;45:612-622 \\
\hline DOI: 10.1159/000509517 & $\begin{array}{l}\text { @ 2020 The Author(s). Published by S. Karger AG, Basel } \\
\text { www.karger.com/kbr }\end{array}$ \\
\hline
\end{tabular}

Cui et al.: Acute Kidney Injury in Patients with COVID-19

5 Bagshaw SM, Laupland KB, Doig CJ, Mortis G, Fick GH, Mucenski M, et al. Prognosis for long-term survival and renal recovery in critically ill patients with severe acute renal failure: a population-based study. Crit Care. 2005;9(6):R700-9.

6 Khwaja A. KDIGO clinical practice guidelines for acute kidney injury. Nephron Clin Pract. 2012;120(4):c17984.

7 National Health Commission of the People's Republic of China [Internet]. Chinese management guideline for COVID-19 (version 7.0) [cited 2020 Mar 4]. Available from: http://www.nhc.gov.cn/yzygj/s7653p/202003/ 46c9294a7dfe4cef80dc7f5912eb1989/files/ce3e6945832a438eaae415350a8ce964.pdf.

8 Vincent JL, Moreno R, Takala J, Willatts S, De Mendonça A, Bruining H, et al. The SOFA (Sepsis-related Organ Failure Assessment) score to describe organ dysfunction/failure. On behalf of the Working Group on SepsisRelated Problems of the European Society of Intensive Care Medicine. Intensive Care Med. 1996 Jul;22(7): 707-10.

9 Wang D, Hu B, Hu C, Zhu F, Liu X, Zhang J, et al. Clinical Characteristics of 138 Hospitalized Patients With 2019 Novel Coronavirus-Infected Pneumonia in Wuhan, China. JAMA. 2020 Feb;323(11):1061.

10 Zhou P, Yang XL, Wang XG, Hu B, Zhang L, Zhang W, et al. A pneumonia outbreak associated with a new coronavirus of probable bat origin. Nature. 2020 Mar;579(7798):270-3.

11 Hoffmann M, Kleine-Weber H, Schroeder S, Krüger N, Herrler T, Erichsen S, et al. SARS-CoV-2 Cell Entry Depends on ACE2 and TMPRSS2 and Is Blocked by a Clinically Proven Protease Inhibitor. Cell. 2020 Apr; 181(2):271-280.e8.

12 Zou X, Chen K, Zou J, Han P, Hao J, Han Z. Single-cell RNA-seq data analysis on the receptor ACE2 expression reveals the potential risk of different human organs vulnerable to 2019-nCoV infection. Front Med. 2020 Apr; 14(2):185-92.

13 Farkash EA, Wilson AM, Jentzen JM. Ultrastructural Evidence for Direct Renal Infection with SARS-CoV-2.J Am Soc Nephrol. 2020 May;ASN.2020040432.

14 Jung JY, Park BH, Hong SB, Koh Y, Suh GY, Jeon K, et al. Acute kidney injury in critically ill patients with pandemic influenza A pneumonia 2009 in Korea: a multicenter study. J Crit Care. 2011 Dec;26(6):577-85.

15 Vincent JL, de Mendonça A, Cantraine F, Moreno R, Takala J, Suter PM, et al. Use of the SOFA score to assess the incidence of organ dysfunction/failure in intensive care units: results of a multicenter, prospective study. Working group on "sepsis-related problems" of the European Society of Intensive Care Medicine. Crit Care Med. 1998 Nov;26(11):1793-800.

16 Cheng Y, Luo R, Wang K, Zhang M, Wang Z, Dong L, et al. Kidney disease is associated with in-hospital death of patients with COVID-19. Kidney Int. 2020 May;97(5):829-38.

17 Chu KH, Tsang WK, Tang CS, Lam MF, Lai FM, To KF, et al. Acute renal impairment in coronavirus-associated severe acute respiratory syndrome. Kidney Int. 2005 Feb;67(2):698-705.

18 Brivet FG, Kleinknecht DJ, Loirat P, Landais PJ; French Study Group on Acute Renal Failure. Acute renal failure in intensive care units-causes, outcome, and prognostic factors of hospital mortality; a prospective, multicenter study. Crit Care Med. 1996 Feb;24(2):192-8.

19 Nin N, Lorente JA, Soto L, Ríos F, Hurtado J, Arancibia F, et al. Acute kidney injury in critically ill patients with 2009 influenza A (H1N1) viral pneumonia: an observational study. Intensive Care Med. 2011 May;37(5):76874.

20 Peiris JS, Chu CM, Cheng VC, Chan KS, Hung IF, Poon LL, et al.; HKU/UCH SARS Study Group. Clinical progression and viral load in a community outbreak of coronavirus-associated SARS pneumonia: a prospective study. Lancet. 2003 May;361(9371):1767-72.

21 Kumar A, Zarychanski R, Pinto R, Cook DJ, Marshall J, Lacroix J, et al.; Canadian Critical Care Trials Group H1N1 Collaborative. Critically ill patients with 2009 influenza A(H1N1) infection in Canada. JAMA. 2009 Nov; 302(17):1872-9.

22 Garzotto F, Ostermann M, Martín-Langerwerf D, Sánchez-Sánchez M, Teng J, Robert R, et al.; DoReMIFA study group. The Dose Response Multicentre Investigation on Fluid Assessment (DoReMIFA) in critically ill patients. Crit Care. 2016 Jun;20(1):196.

23 Wang N, Jiang L, Zhu B, Wen Y, Xi XM; Beijing Acute Kidney Injury Trial (BAKIT) Workgroup. Fluid balance and mortality in critically ill patients with acute kidney injury: a multicenter prospective epidemiological study. Crit Care. 2015 Oct;19(1):371.

24 Chittawatanarat K, Pichaiya T, Chandacham K, Jirapongchareonlap T, Chotirosniramit N. Fluid accumulation threshold measured by acute body weight change after admission in general surgical intensive care units: how much should be concerning? Ther Clin Risk Manag. 2015 Jul;11:1097-106. 